\title{
ALLEGRO: Teaching/Learning Multi-Agent Environment using Instructional Planning and Cases- Based Reasoning (CBR)
}

\author{
Rosa M. Viccari \\ Universidade Federal do Rio Grande do Sul, \\ Artificial Intelligence Group \\ Porto Alegre, Brasil \\ rosa@inf .ufrgs.br \\ Demetrio A. Ovalle \\ Universidad Nacional de Colombia, Computer Science Department, \\ GIDIA: Research and Development Group in Artificial Intelligence \\ Medellín, Colombia \\ dovalle@unal.edu.co \\ Jovani A. Jiménez \\ Universidad Nacional de Colombia, Computer Science Department, \\ GIDIA: Research and Development Group in Artificial Intelligence \\ Medellín, Colombia \\ jajimen1@unal.edu.co
}

\begin{abstract}
This paper presents a description of the environments of individualized learning (Based on the Intelligent Tutoring Systems, ITS), the Computer Supported Collaborative Learning (CSCL), Multi-Agent Systems (MAS) and the artificial intelligence techniques called: Instructional Planning (IP) and Case-Based Reasoning (CBR). Finally ALLEGRO is presented, a MAS environment of support to the teaching/learning process that includes all previous artificial intelligence elements.
\end{abstract}

Keywords: Multi-Agent System, Instructional Planning, Case-Based Reasoning, Artificial Intelligence in Education.

\section{INTRODUCTION}

Day to day, the incursion of computers within the educative context begins to gain importance due mainly to the facility of transmitting knowledge to a range of apprentices. Nevertheless, one of the greater problems in the traditional teaching/learning systems supported by computer is the difficulty to provide an individualized education, adapted to the necessities and specific characteristics of the apprentice. 
The previous problem grows with the explosion of the Internet and the exponential sprouting of these systems which have begun to support distance education through computer or also known as virtual education.

The intention of applying techniques of artificial intelligence to the development of teaching/learning systems supported by computer is to construct more intelligent systems. The term intelligent used in these systems is determined fundamentally by the capacity of adaptation of the instructional environment to the characteristics of learning and knowledge of the different users.

This work looks for focusing the use of computers for teaching/learning purposes. Our motivation is to provide a new way in order to generate Instructional Planning (IP) using the stored experience of cases where the apprentices have solved satisfactorily some problems found during the learning sessions (Case-Based Reasoning, CBR); sometimes, jumping from individual paradigm to the collaborative paradigm. This planning tool is incorporated in a Multi-Agent System (MAS) for integrated surroundings of Intelligent Tutoring Systems (ITS) and Collaborative Environments of Learning Supported by Computer (CSCL); providing autonomy, flexibility and adaptability of the instruction to the apprentice, in this intelligent environment of learning.

Teaching/Learning Environment ALLEGRO is the name of the prototype used to validate pedagogical MAS, where the apprentices can use ITS to receive instruction in an individualized way, and when they consider necessary the collaboration of the other users (teachers, apprentices, monitors, guests, among others) they use the CSCL.

In the following sections is done a review of the individualized and collaborative environments (ITS) of learning supported by computer (CSCL). Section three describes the use of techniques of artificial intelligence in the educative systems supported by computers focusing in IP and CBR. In Section four the approach of MAS is described. In the fifth Section ALLEGRO is introduced, and finally in Section six the conclusions are taught.

\section{INDIVIDUALIZED AND COLLABORATIVE LEARNING EN- VIRONMENTS}

\subsection{Intelligent Tutoring Systems}

Individualized Environments of Learning are based mainly on the Intelligent Tutoring Systems, ITS. They are defined as: "computational systems that are designed to distribute instruction and intelligently support the teaching/learning processes by means of the interaction with the apprentice" [15]. It is called "intelligent" in order to contrast it with the traditional systems of Computer Aided Instruction (CAI) being the difference the use of techniques of artificial intelligence. The ITS have an advantage over the CAI because they can represent the evolution of the apprentice [8].

The ITS represent more advanced pedagogical tools because they provide more experiences of individualized learning, which allows for the education-learning process to be more adaptable to the specific necessities or learning level of each apprentice; this is obtained through the construction and analyses that reflect both the behavior and cognitive aspects [10].

The ITS generate an instructional plan based on the necessities of the apprentice, the Basic Units of Learning, BUL and in the Instructional Objectives, IO. This plan is designed to identify and to define the methods that will help the apprentice to acquire the knowledge; and it is con- 
stantly re-planned according to the results found in the apprentice. The ITS handle some type of experience on the domain that is hoped to be taught, they can reason about the domain, solve problems, evaluate [12], detect errors, offer suggestions, examples, simulations, recommendations and explanations or show the outline of their inferences [19]; just in the same way as a human teacher does it.

\subsubsection{Classification of the ITS}

From a cognitive perspective (Acquisition state of abilities and skills associated to an IO) they can be classified into three generations [11]:

- First Generation: Tutors Based on Restrictions. They are centered in the state of the interface in relation to the information that is shown and/or hidden, graphs, aids, among others.

- Second Generation: Tutors Based on Cognitive Models. They are centered in the actions of the apprentice and the rules that generate from the correct solution of a propose task based on a mental model of the cognitive state (and the work memory) of the apprentice.

- Third Generation: Tutors Based on the Natural Language Dialogue. They are centered in the communication with the apprentice through dialogue.

\subsubsection{Architecture of the ITS}

At present, due to advances in the field of artificial intelligence it can be concluded that there does not exist a unique architecture and that these vary from one implementation to another. The generic architecture includes four modules:

- Tutoring Module: Also known by the name "Instruction and Curriculum". It is in charge of guiding the education-learning process, deciding on what pedagogical actions to make, how and when. It is also in charge of planning and re-planning the instruction according to each apprentice. It chooses the kinds of appropriate problems to be solved, besides to control and criticize the performance of the apprentice [15] or offer stimuli.

- Expert Module: Also known by the name "Module Domain". It is the component that provides the domain with the knowledge to be taught. This knowledge must be organized pedagogically to facilitate the task of the tutoring module [23]. The knowledge can be represented by means of [13] [14]: symbolic rules, fuzzy logic, Bayesian networks, cases-based reasoning, semantic networks, frames, production rules, objects of learning, among others. It has begun recently to represent the knowledge in a hybrid way, that is to say, using two or more formalisms, like for example: neuro-symbolisms and neuro-fuzzy.

- Student Module: It represents the image that the system has about the learning characteristics of the apprentice, administers his individualized information, his behavior [21], limitations, difficulties and motivations, making possible the formulation of hypothesis in order to re-plan the instruction [15] [31]. 
- Interface Module: In charge of handling the interactions between the system and the users (Apprentices, professors, monitors, among others) allowing communication with the modules of the ITS [29].

\subsection{Collaborative Learning Environments Supported by Computer}

The CSCL (Computer-Supported Collaborative Learning) are defined as "the instructional methods aimed at promoting learning through the collaborative effort between apprentices in a certain task of learning, providing an environment that intensifies and enriches the process, where the apprentice interacts with other collaborators in solving a problem" [18]. They are derived from the research field of Computer-Supported Collaborative Work environments (CSCW) referring to a group of people jointly working in the same work environment, causing the collaboration and with the support of the computers. The difference between CSCW and CSCL is in that the latter looks for the apprentices to manage obtaining knowledge in group, instead of looking for efficiency in the work [24].

\subsubsection{Classification}

This technology is classified according to its main objective in the following way [26]:

- Individual Centered: It locally manages the work of each individual within the group.

- Document Centered: It tries hard in managing the tasks assigned to a document, its routing, consulting, updating, among others.

- Process Centered: It controls the conclusion of the activities.

\subsubsection{Architecture of the CSCL}

The architecture of the CSCL also varies from one application to another. The more standardized architecture being the one conformed by four modules [2]:

- Collaborative Support: It is the technology for facilitating communication.

- Pedagogical Strategies: They are based on the learning theories (Theory Located in the Action, to Learn Learning, Learning Based on Problems and Cooperative Learning).

- Experience Characterization: modeling the domain at several levels of abstraction and the identification of strategies for problems solving are its dominant components.

- Knowledge Domain: it refers to the representation of the structure of what is to be taught.

\subsubsection{Types of Applications}

There are the following types of applications [26][3][17]: 
- Face to face interaction: It implies the same time and the same place (Screen shared for explanations, utilities with answer from the audience, conversation surroundings and storms of ideas).

- Centralized Asynchronous Interaction: It implies the same place but different time (Forum of active debate in a computer where people contribute with commentaries).

- Distributed Synchronous Interaction: It implies the same time but different place (distributed synchronous Publishers, work surroundings, chat and videoconference).

- Distributed Asynchronous Interaction: They imply different time and different place (Electronic mail and groups of interest).

\section{ARTIFICIAL INTELLIGENCE TECHNIQUES IN EDUCA- TIVE SYSTEMS}

\subsection{Instructional Planning}

The IP (Instructional Planning) constitutes one of the most important tasks in the educative systems for obtaining the adaptation of the instruction to the apprentice; it is the component in charge of determining the sequence of the actions (Plan) of tutoring in a consistent, coherent and continuous way, which maximizes the learning activities of each apprentice to achieve the IOs during a learning session [22].

The first systems of the years 60 s had a conditional instructional planning prefixed by a teacher. it defined the course by means of a sequence of teaching activities establishing certain points of decision in which, depending on the necessities of the apprentice it was possible to follow one way or another. These plans were expensive to create and difficult to modify. The following step in the evolution was the algorithmic planners in which the sequence of teaching activities is implemented in an algorithm. These algorithms became complex to a great extent in complex domains and were difficult to alter. In order to improve the flexibility of the planners production systems combined with agendas and/or slates were used, with expensive construction and maintenance. The educative programs are able of generating instructional plannings that incorporate the appropriate contents and the necessary learning activities for each apprentice.

\subsubsection{Levels}

Elorriaga [4] proposes an instructional planning model in five levels:

- Basic units of Learning (BULs): It is the sequence that represents the elements in which the knowledge of the domain is organized.

- Instructional Objectives (IOs): They are the cognitive abilities and capacities that the tutor plans for the apprentice to obtain throughout the session.

- Cognitive Processes: Mental activities that must happen in the apprentice. 
- Instructional Events: They are the external conditions that should happen so that learning takes place.

- Instructional Actions: Actions that the tutor and/or the apprentice carry out in the learning sessions.

\subsubsection{Mechanisms}

The IP consists of two parts: the lesson planner and the speech planner. As follows [32]:

- The Lesson Planner: It determines the content of the sequence of the subjects to teach in a simple lesson of learning, it consists as well of two phases: Objectives Generation and Plan Generation. As a result, this planner projects the lessons in a hierarchical way with three sets of rules. The one with the highest level generates the objectives of the lesson and expands one of the objectives within the set of sub-objectives (a plan) for the next level.

- The Planner of the Speech: It is the mechanism for planning the communicative actions between the tutor and the apprentice within a lesson. Some systems communicate with the apprentice in natural language.

\subsection{Reasoning Based on Cases}

The Case-Based Reasoning (CBR) is an artificial intelligence technique that tries to reach the solution of new problems, just as human beings do it using accumulated experience until present in similar events [7]. A new problem is compared with the cases stored previously in the base of cases (Memory of Cases) and one or several of them are recovered. Later a solution is used and evaluated, suggested by the cases that have been selected previously, for trying to apply them to the present problem [28][1].

A case is made up of three elements: the description of the problem, the solution that was applied and the result of the solution. Some CBR use measures to determine which case is best, like for example, the Euclid metric weight fixing values statically for each case using information from experts on the field [27].

The CBR has been applied to a great variety of systems with different intentions: knowledge acquisition, legal reasoning, diagnosis, planning, among others. Systems of teaching/learning have also been developed based on cases; these tutors instruct the apprentice showing to him typical problems as examples or exercises [5].

\subsubsection{Cycle of Performance}

The performance cycle of CBR is formed by 4 processes (Figure 1) [5]:

- Recovering: The present problem is analyzed and their basic characteristics are used to search in the Memory of Cases those that look more similar to the present situation. Among the recovered cases, the most appropriate is selected. 


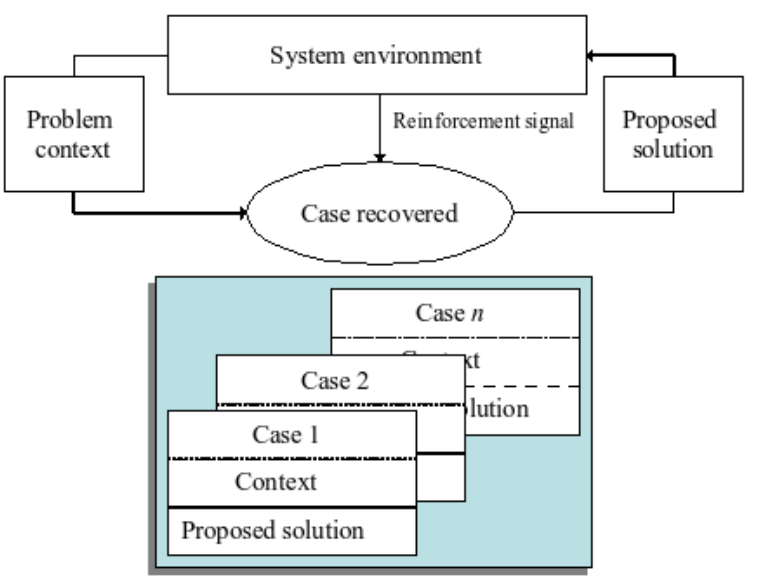

Figure 1: CBR cycle [27].

- Adaptation: Once the case more similar to the present problem has been determined, the system must adapt it so that it adjusts to its peculiarities. The adapted solution is applied to solve the problem in the real domain.

- Reviewing: After applying the solution, the following step consists of reviewing the results obtained in the application to verify whether the proposed solution has been successful or not. If the solution has failed, the causes of the failure are explained and the solution is repaired to avoid it in the future. Therefore, two opportunities of learning occur depending on the evaluation of the results:

- when a problem is solved in a satisfactory way, the data of the experience are used to form a new case (Learning from success).

- when the solution fails, the cause of the failure is identified and the plan is explained and repaired.

- Storage: In this last phase, the system stores in the Memory of Cases the new experience through a case that incorporates the present problem, the solution and its results. If the solution failed, the information necessary to prevent similar failures is stored.

\subsubsection{Reasoning in Computational Teaching/Learning Systems}

The most important are [16]:

- Education Systems Based on Cases: They teach using a set of typical situations. Some teach through telling stories or examples.

- CBR in the Student Module: It has been used in the tasks of cognitive diagnosis and modeling of the apprentice. 
- CBR in the Instructional Decision Process: They make instructional decisions based on cases; some of these systems construct instructional plans for ITS and others produce lessons for teachers.

- Shell of SEBC: They are author tools that allow the teachers to include the domain to be taught and the way in which it must be taught. The system generates session plans in a generic domain and it is able to adapt them to the preferences and objectives of the student.

- Aid tool for teachers: The instructional planning systems are not only used in the context of the education systems but in addition, are useful for the formation of the teachers. For example tools for searching experiences of implantation of educative technology in Internet. These tools are directed to teachers who wish to incorporate educative computer science in their classes allowing them to accede to previous experiences of other teachers in similar educative contexts.

\section{MULTI-AGENT SYSTEMS}

The MAS (Multi-Agent Systems) arise in the decade of the 80s from the software agents researches. The software agents are programs that act in representation of their human users or owners to carry out complicated tasks of information handling, communicate by means of the protocol of passing messages and carrying out their actions concurrently; they have properties like: autonomy, social ability, reactivity, pro-activity, mobility, temporary continuity, adaptability and learning. They live in certain dynamic and complex environments, sensing and acting in an independent way to adapt to the environment; by means of the former, they carry out a series of tasks or goals for which they were designed [10][33].

The software agents can represent pedagogical knowledge and/or carry out tutoring tasks to both support and facilitate the human learning. This type of agents is denominated "Pedagogical Agents" [9]. The pedagogical agents can adapt their instructional interactions to the necessities of the apprentices and to the present state of the learning environment, helping the apprentices in overcoming their difficulties and in profiting from the learning opportunities. They have a set of education objectives, instructional plans for the execution of those objectives, and the resources associated in learning environments. They collaborate with the apprentices and other agents, providing continuous feedback during the working sessions [9].

Going back to the central idea of the present chapter, the MAS are one of the areas of research of Distributed Artificial Intelligence (DAI), the Distributed Problems Solution (DPS) and the Parallel Artificial Intelligence (PAI). A MAS is an organized society composed by semi-autonomous agents that interact with each other, either to collaborate in the solution of a set of problems or in the attainment of a series of individual or collective objectives. These computer science agents can be homogeneous or heterogeneous and can have common or uncommon objectives, but always will involve some degree of communication among them [20]. The principles of the MAS have shown an adequate potential in the development of teaching systems due to the fact that the nature of the teaching-learning problems are more easily solved through a cooperative approach [25]. 


\subsection{Characteristics}

Some of the characteristics that MAS must have are [6]:

- Social Organization: It is the way as the groups of agents organize within the system according to the function or role that corresponds to them, their characteristics, responsibilities, necessities and considering the intention of the communication with the other agents.

- Cooperation: It is the result of sharing intermediate findings to produce an advance in the solution of the particular objectives of other agents and, at the same time contributing to the progressive profit of the global objectives of the system.

- Coordination: By means of communication the agents can coordinate their actions and behavior, having as a result more coherent systems, that is to say, systems that behave well like a unit. The objective of coordination is avoiding situations of conflicts among the agents [30].

- Control: It is the basic support mechanism for the implementation of the coordination mechanisms in MAS. It can be considered from two points of view: global control and local control.

- Communication: The agents can communicate with the purpose of achieving their objectives or those of the society or system in which they live.

\subsection{Teaching/Learning Environments based on MAS}

Some examples are [14]:

- MACES: (Multi-agent Architecture for a Collaborative Educational System). It is a collaborative educative system for distance education. Its architecture is composed by human agents (Apprentices and Tutors) and by five types of artificial agents (Diagnosis, Mediator, Collaborative, Social and Semiotic).

- AMPLIA: (Intelligent Probabilistic Multi-Agent Learning Environment). It is an environment projected like an additional resource for the formation of medicine students. The users (Apprentices, teachers and applications) are represented by autonomous agents that are part of a social meeting based on objectives that communicate, cooperate and negotiate. It has the following artificial agents: Apprentice, Domain and Mediator.

- BAGHERA: It is an educative community of nonhuman agents that interact with each other according to their competences and points of view, cooperating and carrying out educative tasks collectively. The functional objective is to construct a logical experimental, flexible and adaptable platform for distance education. In the environment, each apprentice is supported by three types of agents: Learning Agent Companion (Compagnon-élve), Mediator (Médiateur) and Tutor (Tuteur). Similarly, the teacher is supported by two types of agents: Teaching Companion (Compagnon Enseignant) and Assistant (Assistant).

- MAS-PLANG: The intention is to offer characteristic of adaptability based on styles of learning for supporting distance education through the Web. The environment is made up of 
two levels of agents: those of superior level or personal assistants (Programmable Agent SONIA, Synthetic SMIT, Monitors and Surfing) and those of inferior level or information agents (Didactic Agent and User).

- JADE (Java Agent framework for Distance learning Environments). It offers a set of resources to facilitate the development and implementation of computational environments to be used as instruments of distance education. The agents do not have mobility, they are: Contents Managers, Exercises, Examples, Interactions, Student Model and Communication.

\section{TEACHING/LEARNING MULTI-AGENT ENVIRONMENT ALLEGRO}

ALLEGRO is an intelligent environment conformed by an ITS which allows to offer learning in an individualized way and a CSCL that offers learning in a collaborative way. In order to obtain it, the environment is modeled by means of MAS. The bounties that MAS offers to ALLEGRO are: autonomy, flexibility and adaptability.

When autonomy is mentioned it refers to the initiative taken by the agents of MAS to carry out pedagogical actions without human intervention so that the apprentice achieves the IOs. Also in the way as the system evolves in an independent way in the storage and selection of cases without the necessity of human experts. When flexibility is mentioned it refers to the fact that the apprentice does not only use a methodology for his learning (Individualized/Collaborative). When adaptability is mentioned it refers to the fact that the content of the instruction is molded according to the specific necessities and preferences of the apprentice.

The IP of ALLEGRO uses the CBR technique (Figures 2 and 4), hat is to say, it uses the stored experience of successful solutions of former similar problems. In this sense it is possible to state that the system learns in an independent way from the experience with the apprentices, turning the IP in a flexible tool with the capacity of adapting the knowledge with certain degree of abstraction depending on the apprentice.

The instructional paradigm of ALLEGRO is based on three theories of learning: behaviorist, cognitive and social-historical theory [14].

\subsection{Architecture of the Teaching/Learning Multi-Agent Environment}

The name of the Teaching/Learning MAS Environment ALLEGRO arose from the agreement, harmony and vividness of the elements that integrate it. Its architecture (Figure 2) is conformed by two types of agents (artificial and human) and was implemented in PMA3 (Multi-Agent Platform for Learning Environments) that has as its objective to provide the necessary infra-structure and tool for learning environments based on MAS.

The Teaching/Learning MAS Environment ALLEGRO differs from the other existing environments that approach ITS and CSCL in an implicit way, in their performance, the collection of agent and the profiting from the collaborative channel (specially the asynchronous communication); other differences are [14]: 


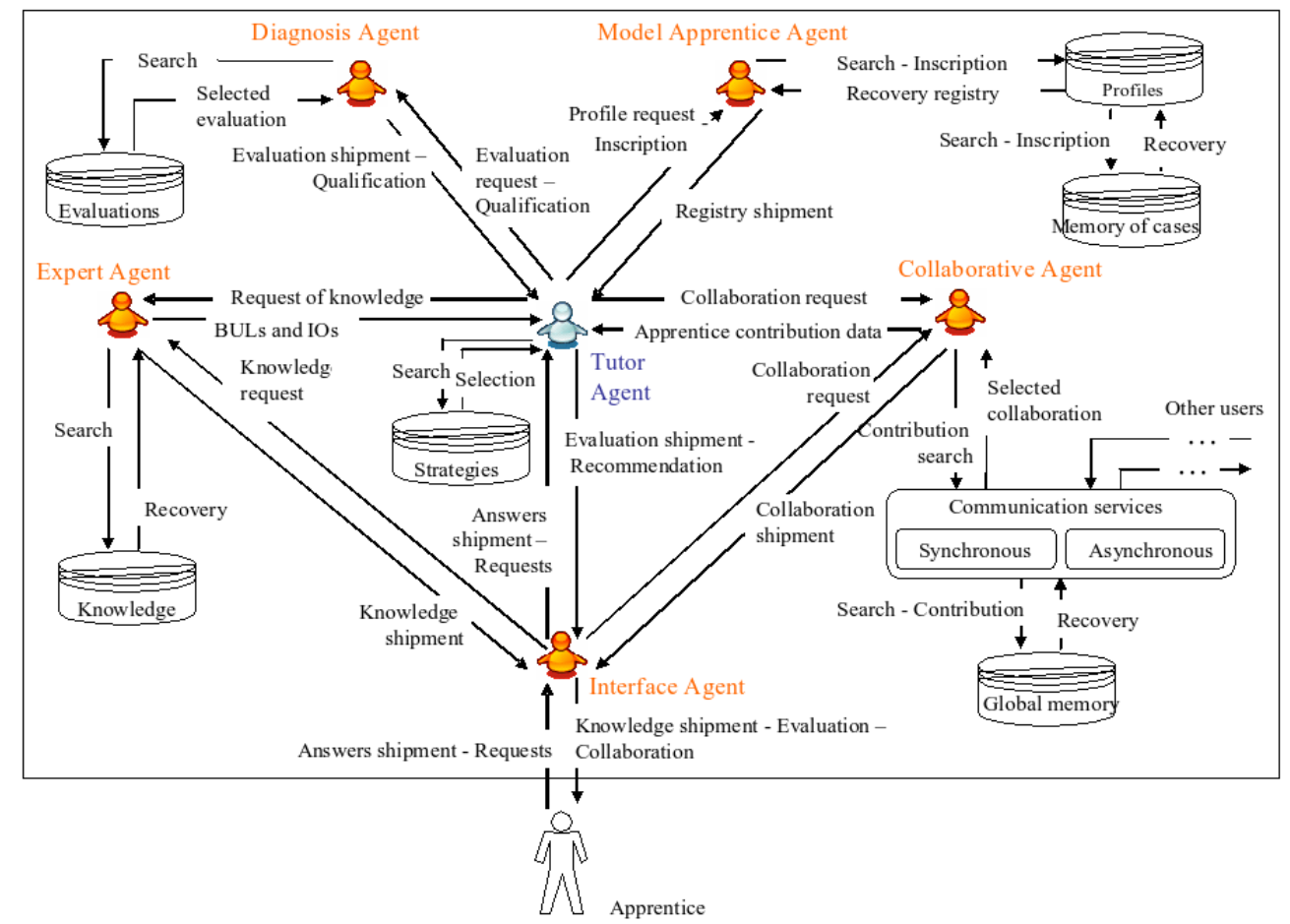

Figure 2: Architecture of the Teaching/Learning Multi-Agent Environment ALLEGRO [14]. 
- MACES, AMPLIA, BAGHERA, MAS-PLANG and JADE do not re-plan the instruction using the stored experience of the apprentices.

- ALLEGRO does not infer emotive states or the affective profile of the apprentice in comparison with MACES. It does not either implement agents animated in comparison with MAS-PLANG.

- The collaborative agent of MACES only uses the synchronous channel of communication in the CSCL. AMPLIA, BAGHERA, MAS-PLANG and JADE do not implement CSCL.

- The representation of knowledge in ALLEGRO is implemented by means of learning objects. AMPLIA implements probabilistic networks. In BAGHERA the knowledge are presented by means of problems to be solved by the apprentice.

Next are shown the functions carried out by each one of the agents in the architecture:

\subsubsection{Artificial Agents}

Are computer science agents in charge of carrying out tasks for their owner within the architecture, in this case pedagogical tasks which are their cognitive component, as well as of showing a certain behavior. There are six types:

- Tutor: Is in charge of guiding the learning process, decides the pedagogical actions to be done, how and when. Their functions are:

- Constantly plan and re-plan the tutoring plan according to the difficulties found and to the profits obtained, that is, according to the specific necessities of the apprentice.

- Ask the Expert Agent, to offer certain knowledge to the apprentice according to the plan and its diagnosis.

- Select the suitable pedagogical strategy, mainly when the apprentice makes a mistake. The strategies allow to better display a content of the domain. Among the pedagogical strategies the learning methodology is shown.

- Ask to the Agent Diagnosis an evaluation for the apprentice according to its profile and IOs.

- Detect mistakes in the process of the apprentice, in addition it offers suggestions, critics and recommendations.

- Apprentice Model: It is in charge of handling the learning model of the apprentice. This model:

- Contemplates the learning style, understanding of the subjects, limitations and knowledge level of the apprentice.

- Maintains individualized information of the apprentice.

- According to the request of the Tutoring Agent, it is in charge of looking for, selecting and passing the case that better adapts to the conditions of the problem (CBR). 
- Interface: It is the bridge between the human and artificial agents. Their functions are:

- Establish and maintain the interaction with the apprentice.

- It allows the unfolding of knowledge and collaboration on the screen of the apprentice.

- Expert: It administers knowledge and contents of the area or specific subject of education. It is made up of BULs and IOs.

- It sends knowledge to the apprentice when he asks for it or at the request of the Tutoring Agent.

- Diagnosis: It is in charge of selecting and grading the knowledge level of the apprentice.

- Collaborative: By request of the Tutoring Agent, it is in charge of looking for other apprentices who are dealing with the same subject and with whom it is possible to establish synchronous or asynchronous communication to offer collaboration to the apprentice.

- It groups the apprentices by study topics, profiles or behavior.

\subsubsection{Human Agents}

These agents can communicate with each other to look for a common objective by means of the division of tasks or to obtain an objective sharing tasks. They are classified in three groups:

- Apprentice: Person who wishes to learn a specific domain. It receives the instructions given by the teacher and the moderator to work in group. It is also known with the names of pupil or student.

- Moderator: It is in charge of managing the learning session is in charge of elaborating and presenting the working agenda; he suggests bibliography or pedagogical resources related to the BUL of study. At the end of a forum he purifies (compiles) the most significant contributions published in the Board, he presents them to the other members by means of a plenary and publishes them as a way of conclusions. The moderator is also known with the names of assistant, auxiliary or monitor.

- Teacher: He is the human teacher, his function is to accompany the apprentices in their formative process, supervising the learning, inspecting the profits and difficulties, with his positive attitude he offers individual and group recommendations, he registers the results of the evaluations. He takes part when a learning session has left the normal trajectory, offers bibliography to the apprentices and to the moderator. He is the one in charge of programming the BULs, as well as the proposed problems.

\subsection{Components of the Teaching/Learning Multi-Agent Environment ALLE- GRO}

\subsubsection{Individualized Environment of Learning}

Is in charge of providing learning in an individual way, just as the ITS do it. It is conformed by five modules (Figure 3): 


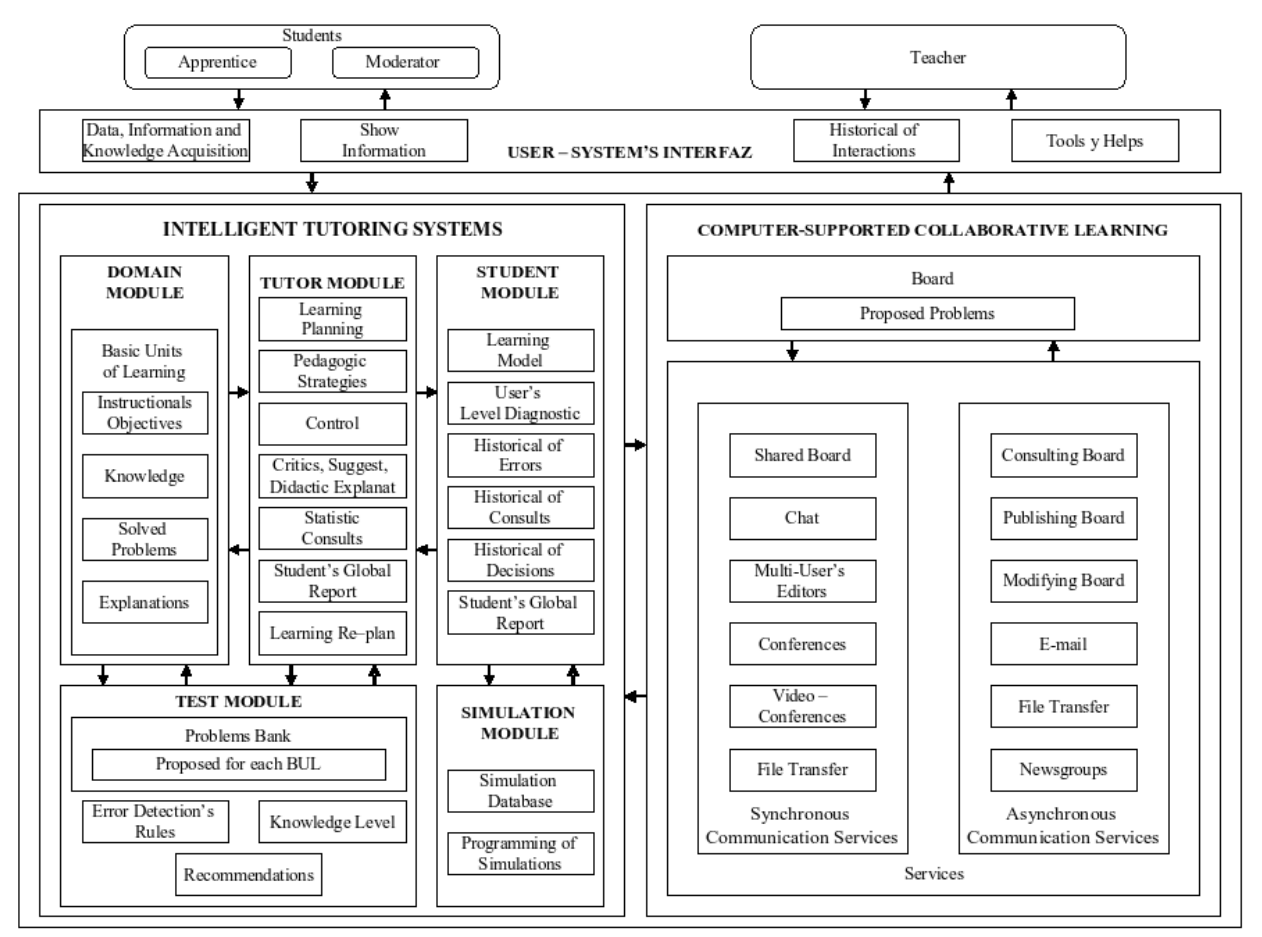

Figure 3: Components of the Teaching/Learning Multi-Agent Environment ALLEGRO.

- Domain Module: It contains and administers the knowledge and contents of the area or specific subject of teaching. It is made up of Basic Units of Learning (BUL) composed by Instructional Objectives (IOs), Knowledge, Problems and Explanations.

- Tutoring Module: It has pedagogical functions and it is in charge of guiding the teaching/learning process, decides what pedagogical actions make, how and when. It is made up of: Learning Planning, Pedagogical Strategies, Control, Didactic Critics, Suggestions and Explanations; Statistical consultations, Global Report of pupils and Learning Re-planning.

- Student Module: It handles the individualized information on each student. It is made up of: Learning Model, Diagnosis Level of the Apprentice, Historical of Errors, Consultations and Decisions; and Global report of students.

- Evaluation Module: Its intention is to measure the state of knowledge of the student according to the advances made. This module is made up of four elements: Bank of Problems - proposed for each BUL-, Rules of Errors Detection, Level of Knowledge and Recommendations.

- Simulation Module: It is in charge of showing the way of solving a problem or physical process. It has two elements: Data base of Simulations and Programming. 


\subsubsection{Learning Collaborative Environment}

Is in charge of promoting learning through the collaborative effort among the users in the learning sessions and it is made up of (Figure 3):

- Board-Proposed Problems. It keeps stored the bank of proposed problems, the agenda and contributions that the apprentices offer for solving these problems.

- Services. They are components that allow the direct or indirect handling of communication among users, there are two types: synchronous and asynchronous communication.

\subsection{IP Model using CBR of the Teaching/Learning Multi-Agent Environment}

The IP model using CBR is innovative in the sense that it uses the services of synchronous and/or asynchronous communication proper of the CSCL, in addition it is equipped with autonomy and flexibility thanks to the attributes of the artificial agents.

The essential task of IP using CBR is carried out by the artificial agents Tutoring and Model of the Apprentice, the other software agents serve as support to the teaching/learning process. If an apprentice has a non satisfactory result when finalizing the evaluation of a BUL, the system activates the context of the CBR searching and selecting a successful case in which another apprentice after having difficulties achieved the proposed IOs for the BUL in study. Then it re-plans the whole context so that the apprentice tries again to satisfactorily obtain the BUL by means of the selected case (Figure 4). The IP model has four elements: Planner, Executor, Evaluator and Recovery. Next the process is detailed step by step:

- The process begins when the apprentice enters the system (Login) using his identification. The data are received and validated by the Interface agent and sent to the agent Model of Apprentice to identify the students profile, this determines the BUL and IOs to be learned. This information is sent to the Tutoring agent to elaborate the instructional plan (Planner).

- The component Executor sends to the apprentice the knowledge and collaboration that he requires, by means of the coordination among the Expert, Collaborative and Interface Agents according to the actions planned by the Tutoring agent in the previous state.

- The following step in the instructional process is to diagnose the level of knowledge acquired by the apprentice through an evaluation of the IOs which correspond to the BUL. This task is carried out by the Diagnosis agent, just as the respective evaluation. If the result of the evaluation satisfies the acquisition of the IOs, this information is sent to the agent Model of the Apprentice in order to update and to save the profile of the student. The system allows the student to pass to the study of the following BUL within the instructional cycle.

- When the results of the evaluation do not satisfy the IOs, the Tutoring agent and Model of the Apprentice call the Recovery component which looks for and selects a similar case where the apprentices with a similar profile have solved a similar situation and have achieved the IOs. The recovered case is proposed as a solution and sent to the Planner component (Tutoring Agent) to make the changes in the previous plan (Re-planning the instruction) within the instructional cycle. 


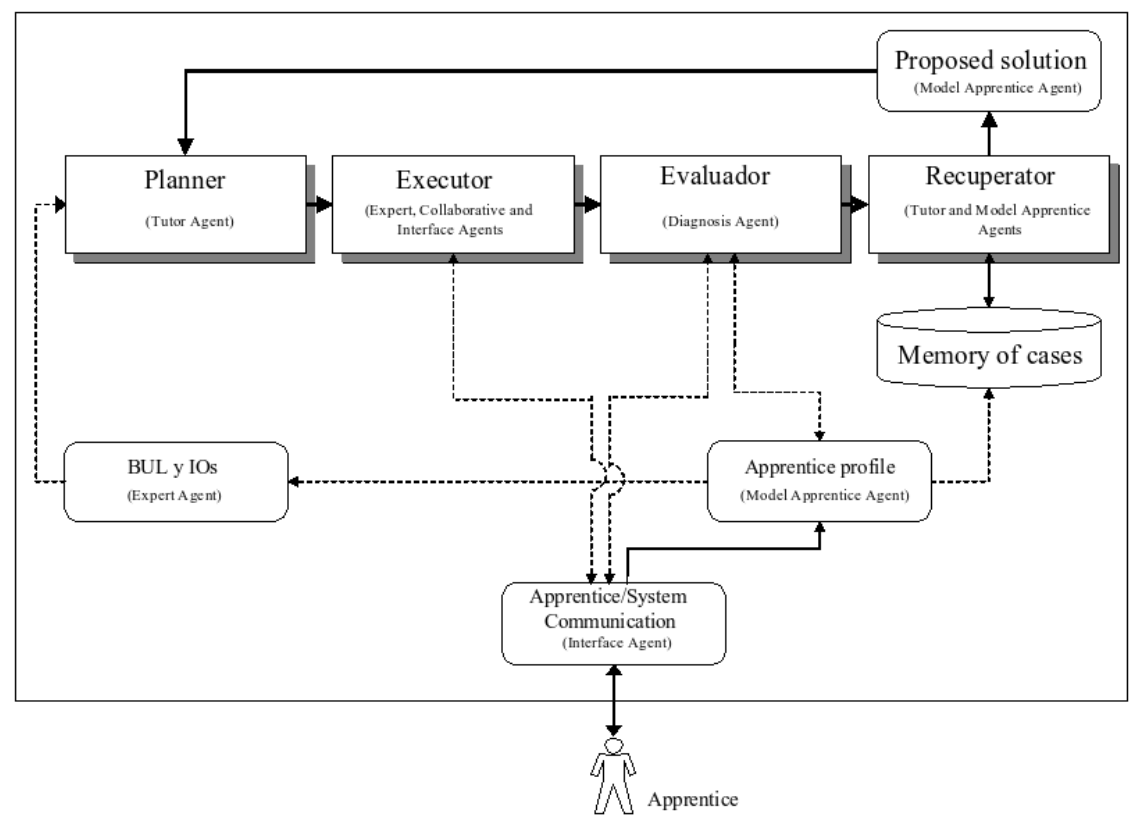

Figure 4: IP using CBR implemented in ALLEGRO [14].

- When the apprentice has obtained satisfactorily the IOs after using the memory of cases, the information is updated and recorded in the profile of the student and the memory of cases to be used in future sessions of teaching/learning by other apprentices.

- When the apprentice does not achieve the IOs and the Recovery component does not find a case similar, the Planner component (Tutoring Agent) sends a requirement to the Collaborative agent so that it suggests and establish communication with other more advanced students in the topic that are found in the collaborative channel (synchronous or asynchronous) in order to help the apprentice. After achieving the IOs, the new case is saved in the memory of cases.

\subsection{Description of the Cases in the ALLEGRO IP}

The structure of each one of the cases (Figure 5) is composed by five fields: Identification of the Case, Context, Solution, Euclidian Weight and Result Obtained after applying the case. Next they are detailed:

- Case Identification: It is a consecutive number that allows distinguishing of the stored cases.

- Context: Made up by the name of the BUL, the IOs and the learning level of the student. This last field is used by the Tutoring agent to ask to the Expert agent for knowledge and problems with certain degree of abstraction. 


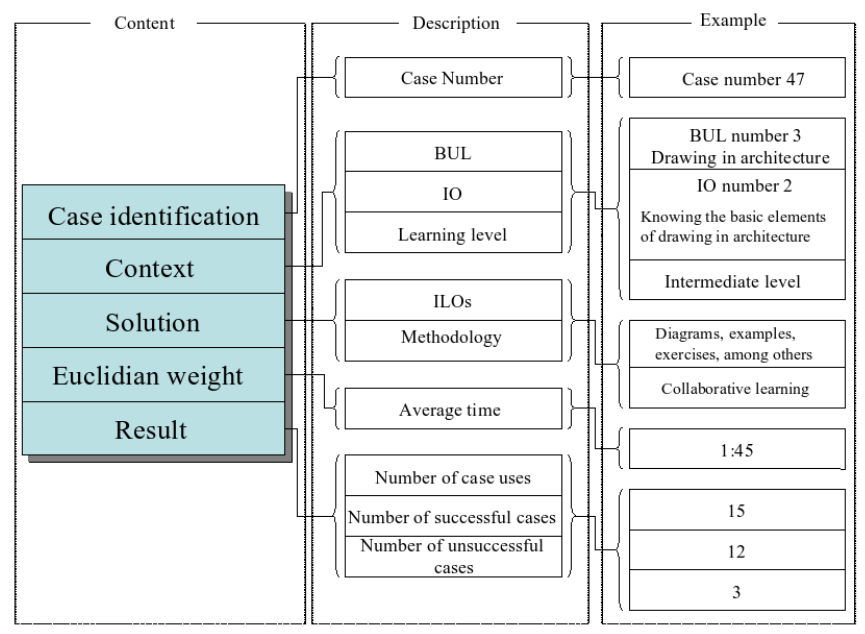

Figure 5: Cases structure [14].

- Solution: It shows objects of specific learning (Knowledge) and the working methodology which has been used by the other apprentices.

- Euclidian Weight: It is calculated by means of the arithmetic average of the time used by the apprentices in achieving the IOs when they use certain case.

- Result: It contains the number of times that the case has been used by the apprentices, the number of successful cases when using the specific case and the number of non successful cases.

\section{CONCLUSIONS}

At present great changes are taking place in the way in which people live, learn and teach; due specially to the impressive advances in the field of computer science and telecommunications, which represents a new challenge for the researchers in the area of artificial intelligence in education.

The IP constitutes one of the main and more complicated tasks of the teaching/learning systems. Traditional mechanisms of planning are based on explicit models whose construction and maintenance are, generally, difficult and expensive. Nevertheless, if they are equipped with the learning capacity it facilitates the construction, equally broadening, its capacity of adaptation to different types of apprentices, and even in different domains.

The CBR is an appropriate mechanism to solve problems in computational systems of teaching/learning, thanks to its capacity to reflect on the results obtained and to store the experiences.

The Teaching/Learning Multi-Agent Environment ALLEGRO represents a set of important achievements in the area of artificial intelligence in the education affecting favorably the learning of the students because it allows to offer them education in an individualized way just as ITS do it and at the moment that the apprentice considers necessary the collaboration of other members 
or when he wishes to collaborate to them, he uses the CSCL, causing in this way the formation of knowledge in group.

It becomes necessary to mention the resistance of some teachers when facing new ways of instruction for implementing the researches and developments in the area of artificial intelligence in the education. The innovation of the teaching/learning traditional methods is urgently necessary, but before that a work of awareness and computational formation among teachers must be carried out.

When establishing the importance of artificial intelligence in the education for the construction of new ways and in the generation of new attitudes among the protagonists of the teaching act, it is understood the meaning of the present work.

\section{ACKNOWLEDGEMENTS}

The work described in this paper is supported by a COLCIENCIAS scholarship within the framework of the Program of Support to the National Scientific Community through the 2003 Doctorate Programs. Also it was partially financed by the research project entitled: "Model of Multi-Agent System of Adaptive Courses integrated with Collaborative Learning Environments" of the Vicerectory Research of the Universidad Nacional de Colombia.

\section{References}

[1] Chua D and Loh P. CB-Contract: Case-Based Reasoning Approach to Construction Contract Strategy Formulation. Journal of Computing in Civil Engineering, 20(5), 2006.

[2] Dorneich M. A System Design Framework-Driven Implementation of a Learning Collaboratory. IEEE Transactions on Systems, Man and Cybernetics - Part A: Systems and Humans, 32(2), 2002.

[3] Ellis C, Gibbs S, and Rein G. Groupware Some Issues and Experiences. Communications of the ACM, 34(1), 1991.

[4] Elorriaga J. Planificación de la Instrucción en Sistemas Tutoriales Inteligentes Evolutivos desde un Enfoque de Razonamiento Basado en Casos. PhD thesis, Universidad del País Vasco, 1998.

[5] Elorriaga J and Fernandez-Castro I. Using Case-Based Reasoning in Instructional Planning. Toward a Hybrid Self-improving Instructional Planner. International Journal of Artificial Intelligence in Education, 11, 2000.

[6] Ferber J. Multi-Agent Systems: An Introduction to Distributed Artificial Intelligence. 1999.

[7] Fowler M. UML Destilled. 2 edition, 2000.

[8] Gaudioso E. Contribuciones al Modelado del Usuario en Entornos Adaptativos de Aprendizaje y Colaboración a través de Internet mediante Técnicas de Aprendizaje Automático. $\mathrm{PhD}$ thesis, Universidad Complutense de Madrid, 2002. 
[9] Giraffa L and Viccari R. The Use of Agents Techniques on Intelligent Tutoring Systems. International Conference of Chilean Society of Computer Science, Proceedings: IEEE Computer Society, 1998.

[10] Gomes E, Boff E, and Viccari R. Social, Affective, and Pedagogical Agents for the Recommendation of Tutorial Colleagues in Agent Based Learning Environments. In Proceedings of Workshop Social and Emotional Intelligence in Learning Environments, 7th International Conference Intelligent Tutoring Systems, ITS2004, Brasil, 2004.

[11] González C. Intelligent System in Education: A Review of Current Research Lines. E-Journal of Educational Research, Assessment and Evaluation, 1(1), 2004.

[12] Guzmán E and Conejo R. A Model for Student Knowledge Diagnosis through Adaptative Testing. In Proceedings of 7th International Conference Intelligent Tutoring Systems, ITS2004 Brasil, 2004.

[13] Hatzilygeroudis I and Prentza J. Knowledge Representation Requirements for Intelligent Tutoring Systems. In Proceedings of 7th International Conference Intelligent Tutoring Systems, ITS2004, Brasil, 2004.

[14] Jiménez J. Un Modelo de Planificación Instruccional usando Razonamiento Basado en Casos en Sistemas Multi-Agente para entornos integrados de Sistemas Tutoriales Inteligentes y Ambientes Colaborativos de Aprendizaje. PhD thesis, Universidad Nacional de Colombia, 2006.

[15] Jiménez Jovani and Ovalle Demetrio. Entorno Integrado de Enseñanza/Aprendizaje basado en ITS \& CSCL. Revista Iberoamericana de Sistemas, Cibernética e Informática, USA, 1(1), 2004.

[16] Khan T and Yip Y. CBT II - Cased-Based Computer-Aided Instruction: Survey of Principles, Applications and Issues. The Knowledge Engineering Review, 10(3), 1995.

[17] Kosoresow A and Kaiser G. Using Agents to enable Collaborative Work. IEEE Internet Computing, 1998.

[18] Kumar V. Computer-Supported Collaborative Learning: Issues for Research. Technical report, University of Saskatchewan, Saskatoon, Canada, May 2005. http://www.sfu.ca/ vivek/personal/papers/CSCLIssuesForResearch.pdf.

[19] Lelouche R and Thoan T. Using a Framework in the Development of an Intelligent Tutoring System. IEEE International Conference on Information Reuse and Integration - IRI03, 2003.

[20] Lemaitre Ch. Multi-Agent Network for Cooperative Work. Expert System with Applications: An International Journal Elsevier Science, 1998.

[21] Limoanco T and Sison R. Learner Agents as Student Modeling: Design and Analysis. In Proceedings of 3rd IEEE International Conference on Advanced Learning Technologies ICALT2003, 2003. 
[22] Matsuda N and VanLehn K. Decision Theoretic Instructional Planner for Intelligent Tutoring Systems. In Proceedings of Workshop on Modeling Human Teaching Tactics and Strategies, 2000 .

[23] Ming G and Quek C. EpiList: An Intelligent Tutoring System Shell for Implicit Development of Generic Cognitive Skills That Support Bottom-Up Knowledge Construction. IEEE Transactions on Systems, Man \& Cybernetics: Part A, 37(1), 2007.

[24] Okamoto T, Kayama M, and Cristea A. Proposal for Collaborative Learning Standardization. In Proceedings of IEEE International Conference on Advanced Learning Techniques ICALT01, 2001.

[25] Oliveira F and Viccari R. Are Learning Systems Distributed or Social Systems. In Proceedings of European Conference on AI in Education, 1996.

[26] Ortega M and Bravo J. Groupware and Computer-Supported Collaborative Learning, 1998.

[27] Paulson P and Juell P. Reinforcement Learning in Case-Based Systems. IEEE Potentials, 4, 2004.

[28] Reyes R and Sison R. Case Retrieval in CBR-Tutor. In Proceeding of International Conference on Computer in Education - ICCE02 IEEE, 2002.

[29] Suebnukern S and Haddawy P. A Collaborative Intelligent Tutoring System for Medical Problem-Based Learning. Proceedings of 9th International Conference on Intelligent User Interface, 2004.

[30] Thibaud M, Daniel R, and Didier A. Multi-Site Coordination using a Multi-Agent System. Computers in Industry, 58(4), 2007.

[31] Virvou M, Manos K, and Katsionis G. An Evaluation Agent that Simulates Students Behavior in Intelligent Tutoring Systems. IEEE International Conference on Systems, Man and Cybernetics - SMC03, 5, 2003.

[32] Woo C. Instructional Planning in an Intelligent Tutoring System: Combining Global Lesson Plan with Discourse Control. PhD thesis, Illinois University, 1991.

[33] Wooldridge M. An Introduction to Multi-Agent Systems. 2002. 